\title{
STUDY OF WAVE IMPACT ONTO A SEMI-SUBMERSIBLE PLATFORM USING MOVING PARTICLES SEMI-IMPLICIT (MPS) METHOD
}

\author{
C. A. Bellezi ${ }^{1}$, L. Y. Cheng ${ }^{2}$ \\ ${ }^{1}$ Department of Naval Architecture and Oceanic Engineering, Escola Politécnica, University \\ of São Paulo (cbellezi@tpn.usp.br) \\ ${ }^{2}$ Department of Construction Engineering, Escola Politécnica, University of Sao Paulo
}

\begin{abstract}
This paper presents a study on the complex phenomenon of fluid-structure interaction involving the wave impact onto semi-submersible platform by using Moving Particles Semi-Implicit (MPS) method. The MPS method is a fully lagrangian particle-based which is originally developed to model incompressible flows and is promising in highly non-linear phenomena analysis, such as the hydrodynamic impact with large free surface deformation and fragmentation. Is this study hydrodynamics of wave in deck impact and wave runup in two different type of semi-submersible platforms, with square section columns and with circular section column are considered. Also, the effects of the motion of the platforms of the phenomena are analyzed by comparing the responses when the platform is fixed and is free floating with coupled heavy and pitching motions.
\end{abstract}

Keywords: fluid-structure interaction, particle method, semi-submersible platform, wave impact, offshore numerical tank

\section{INTRODUCTION}

The recent discovery and exploration of Brazilian pre-salt oil reservoir demands researches and developments focused on oil exploitation in deep water and ultra deep water. In this type of operation, floating structures such as semi-submersible platforms and FPSO (floating, production, storage and offloading) are usually adopted, and suffer the effects of the environmental loads due to winds, waves and currents.

The impact of waves onto offshore platforms is associated with a series of phenomena which may affect the operation or cause serious damage to plant and structures. Among these phenomena, wave-in-deck impact occurs when the wave height is greater than the air gap and the wave reaches the bottom of the deck of a semi-submersible, and imposes high loads for which the structures usually are not projected to suport. An experimental study of the phenomenon of wave-in-deck impact is presented in [1], comparing the effects of a bi-dimensional modeling and a three-dimensional modeling of the phenomena. Experimental and a numerical stud- 
ies using a finite volume method are presented in [2], which concluded that the suction force at the wave output phase has the same magnitude of the initial wave impact over the deck. In [3] the experimental results from [1] and the numerical results obtained from finite volume method [2] are compared to that obtained from the particle method based on Moving Particle Semi-implicit (MPS) method. The particle method results of the impact force horizontal component and the runup are closer to the experimental data than the mesh method. However the MPS method does not calculate the negative pressures during the output wave phase.

Also associated with severe weather conditions and large amplitude waves are the green water phenomenon (also called shipping water), that consists of the water invasion on the deck, damaging plant equipments. The phenomenon of green water is common at FPSO platforms, due to the hull freeboard restrictions, but can occur in semi-submersible when the wave height is large enough. In [4] and [5], the MPS method was applied to study green water on shipping vessels.

Another phenomenon is the wave runup, the wave height amplification during the wave impact onto structures and may exert severe structural loads on the columns of semi-submersible platform. The focus of [6] is the analysis of the wave runup onto semi-submersible platform columns comparing experimental data with numerical results using a finite volume method.

The wave impacts are very complex and highly nonlinear problems. In this study, for the modeling of incompressible flows with large free-surface deformation and fragmentation, Moving Particles Semi-Implicit (MPS) method proposed by [7] is used. The method adopts fully lagrangian description of computational domain and the governing equations differential operators are derived from a particle-interaction model. In relation to the analytical methods and the eulerian description and mesh-based computational methods, MPS is promising in the treatment of highly nonlinear phenomena.

In this study numerical simulations of wave impact on semi-submersible platforms are carried out in a numerical towing tank. The first stage of the study consists on the wave calibration of the incoming wave. The second stage consists on the wave impact over the floating structure in head seas with coupled pitch and heave motions. The future development of this work is focused on the use of higher resolution models with six degrees of freedom.

\section{MPS PARTICLE METHOD}

\subsection{Governing Equations}

The incompressible flow governing equations are the continuity equation (Eq. (1)) and the Navier-Stokes equation (Eq. (2)).

$$
\frac{D \rho}{D t}=-\rho(\nabla \cdot \vec{u})=0
$$




$$
\frac{D \vec{u}}{D t}=\frac{1}{\rho} \nabla \mathrm{P}+\vartheta \nabla^{2} \vec{u}+\frac{\vec{f}}{\rho}+\vec{g}
$$

Where $\rho$ is the fluid density, $\vec{u}$ the velocity vector, $P$ the pressure, $\vec{g}$ the gravitational aceleration, $\vec{f}$ the external forces, $\vartheta$ the dynamic viscosity and $t$ the time.

\subsection{Particle Interation Model}

In MPS method, the differential operators are replaced by operators derived by a particle interaction model based on the contributions weighting of each particle inside a neighborhood radius. The weight function is:

$$
\omega(r)=\left\{\begin{array}{c}
\frac{r_{e}}{r}-1 \text { para }\left(0 \leq r<r_{e}\right) \\
0 \text { para }\left(r_{e} \leq r\right)
\end{array}\right.
$$

In which $r=\left|\vec{r}_{j}-\vec{r}_{l}\right|$ is the distance between two particles $i$ and $j$ and $r_{e}$ the neighborhood radius. The particle number density (pnd), is shown in equation (4). The pnd is proportional to the fluid density and a constant $p n d$ value satisfys the incompressibility.

$$
[p n d]_{i}=\sum_{j \neq i} \omega\left(\left|\overrightarrow{r_{j}}-\overrightarrow{r_{i}}\right|\right)
$$

The gradient operator (Eq. (5)) and the laplacian operator (Eq. (6)) of a scalar function $\Phi$ are presented below:

$$
\begin{gathered}
{[\nabla \Phi]_{i}=\frac{d}{p n d^{0}} \sum_{j \neq i}\left[\frac{\left(\Phi_{j}-\Phi_{i}^{\prime}\right)}{\left|\vec{r}_{j}-\vec{r}_{l}\right|^{2}}\left(\vec{r}_{j}-\vec{r}_{l}\right) \omega\left(\left|\vec{r}_{j}-\vec{r}_{l}\right|\right)\right]} \\
{\left[\nabla^{2} \Phi\right]_{i}=\frac{2 d}{p n d^{0} \delta} \sum_{j \neq i}\left[\left(\Phi_{j}-\Phi_{i}\right) \omega\left(\left|\vec{r}_{j}-\vec{r}_{l}\right|\right)\right]}
\end{gathered}
$$

Where the constant $\delta$ is given by:

$$
\delta=\frac{\int_{V} \omega(r) r^{2} d v}{\int_{V} \omega(r) d v}
$$

Where $d$ is the number of dimensions and $p n d^{0}$ is the initial particle number density value. 


\subsection{Free-Surface Boundary Condition}

For a particle $i$, if its particle number density satisfies $p n d_{i}<\beta p n d^{0}$, then it is considered as a free-surface particles and atmosphere pressure is imposed as its pressure. Usually $\beta=0,97$ is used.

\section{4. Incompressible Flow Algorithm}

The algorithm of MPS method is divided into two parts. The first consists on the explicit calculation of velocity, position and pnd of the particles using the Navier-Stokes equations considering all the terms on the right side of the equation, except the pressure gradient. The second stage is the implicit calculation of pressure solving a linear system of Poisson equation (Eq. (8)) derived from mass conservation.

$$
\left[\nabla^{2} P^{n+1}\right]_{i}=\frac{\rho}{\Delta t^{2}} \frac{\left[p n d^{*}\right]_{i}-p n d^{0}}{p n d^{0}}
$$

Where $p n d^{*}$ is the pnd estimated over the calculation explicit stage. And finally, the correction of velocity and position are done. The MPS algorithm is presented in the Figure 1 flowchart.

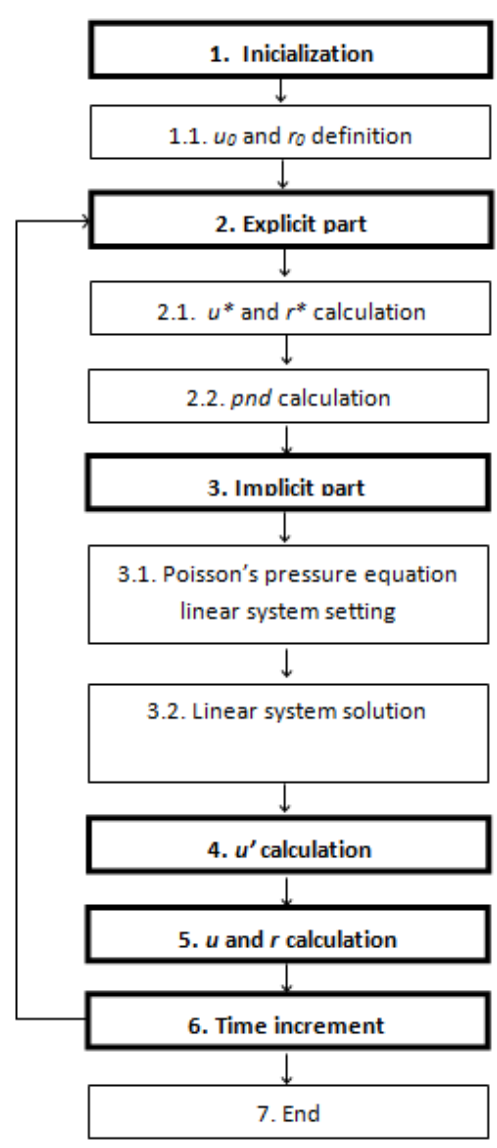

Figure 1. MPS algorithm flowchart 


\subsection{Fluid-Structure Interaction}

In MPS method, the solid boundaries are also discrete in particles. In order to calculate correctly the pnd of the particles near the boundaries, two additional layers of dummy particles are used (Figure 2).

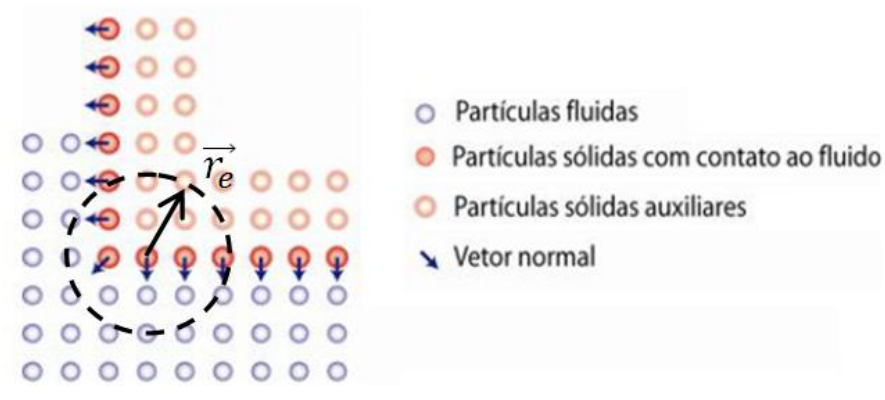

Figure 2. Solid particles and solid dummy particles

In the present, three different solid types are used: the fixed solid; solid with forced motion and the free motion solid. The fixed solid has zero velocity. In the solid with forced motion solid the particle velocity is imposed during the explicit calculation. The free motion solid moves under the influence of external forces and moments. The free solid implementation used in this study is based on [8] and [9], which also presents several cases of validation. 


\section{WAVE CALIBRATION}

The incoming waves characteristics are based on [4]. Three different kinds of waves are simulated: a short wave, a medium wave and a long wave; all with high amplitudes simulating severe weather conditions. Table 1 show the characteristics of the three waves at real scale and the 1:50 model scale.

Table 1. Wave characteristics

\begin{tabular}{ccccc}
\hline Model scale & \multicolumn{2}{c}{$1: 1$} & \multicolumn{2}{c}{$1: 50$} \\
\hline Wave type & $\mathrm{H}[\mathrm{m}]$ & $\mathrm{T}[\mathrm{s}]$ & $\mathrm{H}[\mathrm{m}]$ & $\mathrm{T}[\mathrm{s}]$ \\
\hline Short & 7,6 & 9,0 & 0,152 & 1,29 \\
Medium & 15,0 & 11,0 & 0,3 & 1,6 \\
Long & 20,5 & 13,0 & 0,4 & 1,89 \\
\hline
\end{tabular}

The first stage of the research comprises the incoming wave calibration based on the wave height and the period (or the wavelength) of desired waveform. In this work a validation study of the flap type wavemaker for the MPS simulation code was performed, with similar procedure to that presented in [10], in which the numerical results and the analytical ones based on the wavemaker theory [11] were compared for validation of the wavemaker. The movement amplitude range, the period and tank depth influences over the wave characteristics was also investigated.

In this study, the fluid is treated as inviscid and has $1000.0 \mathrm{~kg} / \mathrm{m}^{3}$ density. Figure 4 shows the wave profile alog the numerical tank. The horizontal axis is the distance from the wavemaker and vertical axis is the wave elevation. As shown in Figure 4-a, the amplitude of the wave generated numerically reduces gradually as it advances to the opposite end of the tank. This reduction is caused by numerical damping and, therefore, in addition to the period and amplitude, the point of the tank in which the generated wave has the requested wave height must be found. This point is defined as frame position $\left(P_{f r}\right)$, where the model will be placed. Figure 4-b shows the wave amplitude reduction.

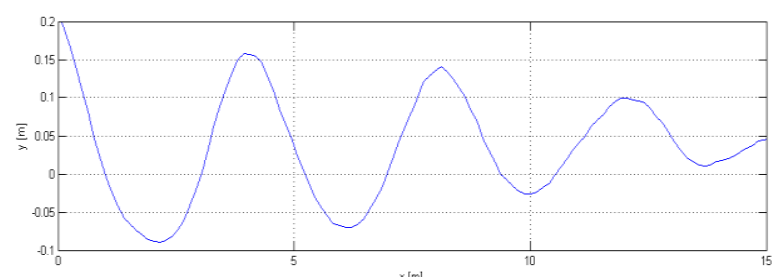

(a)

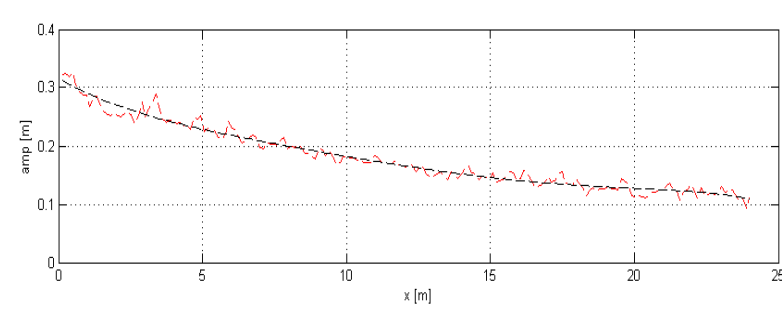

(b)

Figure 4. (a) Free surface wave profile (b) Waveheight reduction along the tank - Medium wave 
The tank used in the numerical wave calibration tests has 1 meter depth and 20 meter length. Table 2 shows the calibrated waves characteristics, the frame position and flap type wavemaker motion amplitude (in radians) for 1:50 scaled model.

Table 2. Wave characteristics - MPS simulation

\begin{tabular}{ccccc}
\hline Wave type & $\mathrm{H}[\mathrm{m}]$ & $\mathrm{T}[\mathrm{s}]$ & $\mathrm{P}_{\mathrm{fr}}[\mathrm{m}]$ & $\theta[\mathrm{rad}]$ \\
\hline Short & 0,16 & 1,29 & 0,76 & 0,1 \\
Medium & 0,32 & 1,6 & 2,36 & 0,25 \\
Long & 0,41 & 1,89 & 2,96 & 0,4 \\
\hline
\end{tabular}

Figure 5 shows free surface elevation time series for the three wave types at the frame positions. As the position of the free surface presents some scattering, the time series presented are filtered. As shown in Figure 5, the generated wave presents asymmetric height of crests and hollows, which is typical of the shallow water waves.

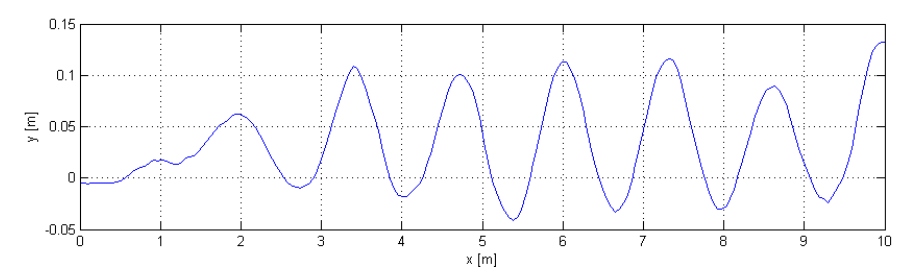

(a)

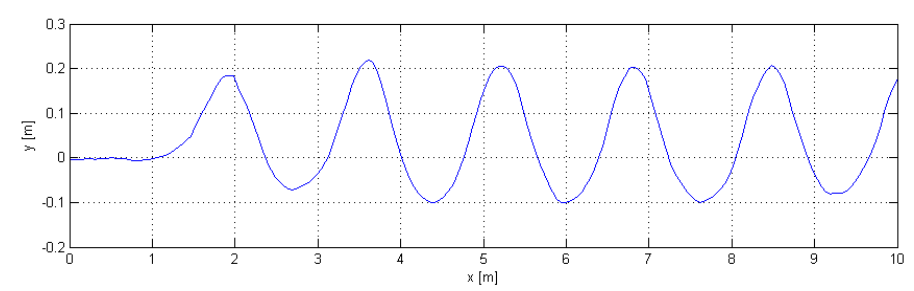

(b)

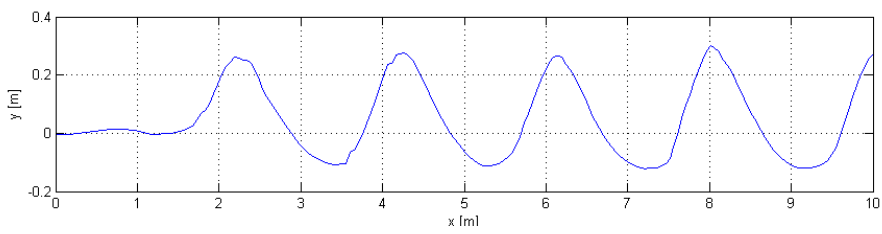

(c)

Figure 5. Frame Position wave elevation time series $\left(P_{f r}\right)$ (filtered results) - (a) Short wave (b) Medium wave (c) Long wave 


\section{MODELS}

The simulations of the wave impact are carried out by using two 1:50 scaled models, which differ in the shape of columns, one with square section columns and the other with circular section columns. The full-scale structures dimensions are shown in Figure 6 and Figure 7. The draft of the structures is 16 meters in full scale.
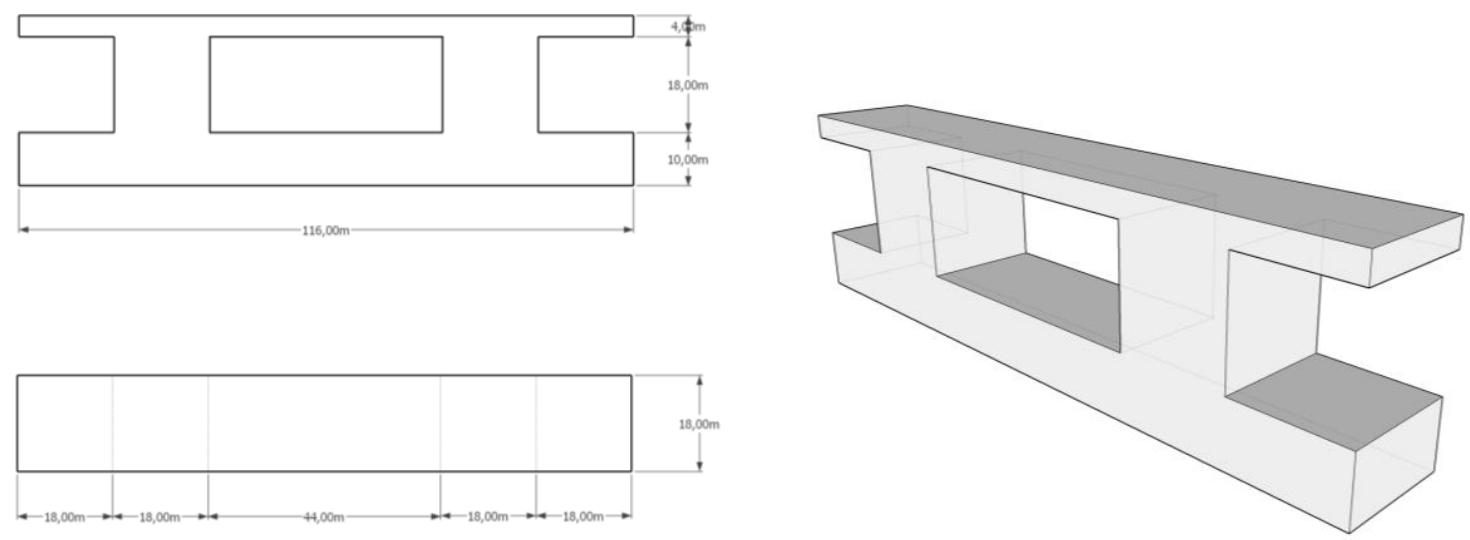

Figure 6. Square section column structure dimensions
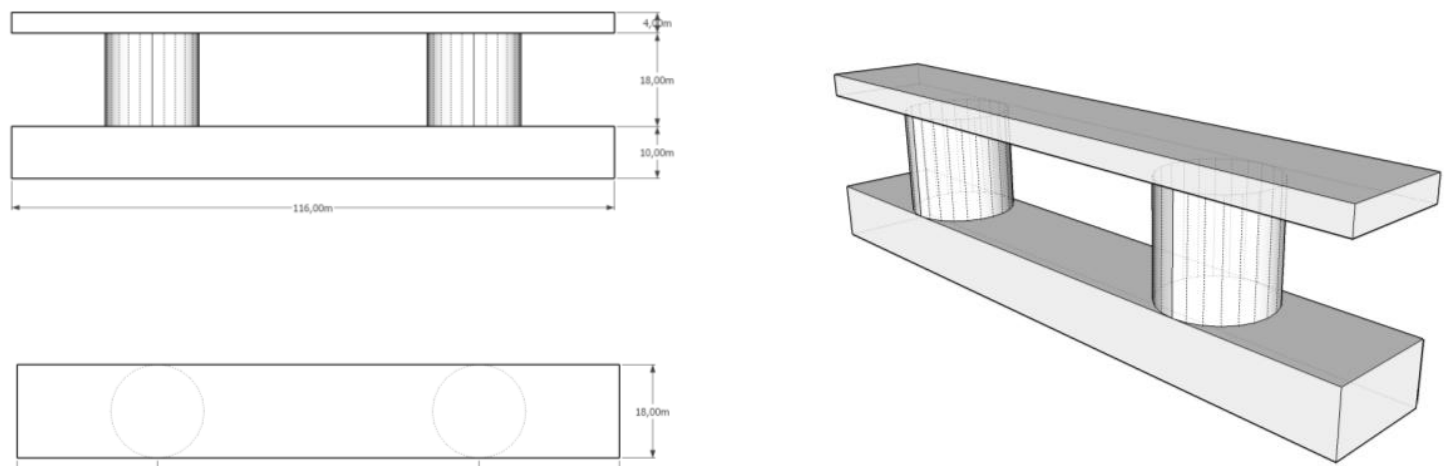

Figure 7. Circular section column structure dimensions

The models presented in Figure 6 are based on the dimensions of the one used in [6]. In which numerical results obtained by an improved finite volume method are compared to the experimental data from a 1:50 scale model.

The width of the numerical tank is five times the structure midsection width. At the front wall of the tank is positioned a flap wavemaker, modeled as a forced movement solid. At the end of the tank is positioned 45 degrees sloped beach. The tanks used in the simula- 
tion of three different wave types have different lengths according to the frame position. Figure 8 shows the numerical test tank configuration.

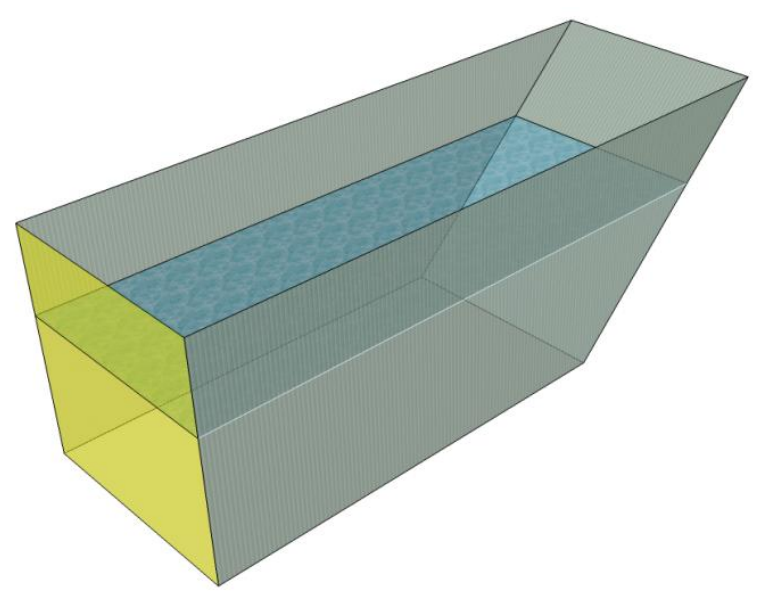

Figure 8. Numerical test tank

One of the main challenges of the MPS method is the fluctuations in the value of the calculated pressure. To mitigate this shortcoming, a technique from [12] is applied in the present study, by adding a smoothing constant at Poisson's pressure equation (called pressure smooth coefficient) and by the careful choice of the numerical simulation parameters. The simulation numerical parameters are shown in Table 3.

Table 3. Numerical simulation parameters

\begin{tabular}{ccc}
\hline $\begin{array}{c}\text { Distance between } \\
\text { particles }[\mathrm{m}]\end{array}$ & Time step [s] & $\begin{array}{c}\text { Pressure smooth coef- } \\
\text { ficient }\end{array}$ \\
\hline 0,02 & 0,00075 & 0,075
\end{tabular}

For the simulations of free motion floating structure, mass, the mass center position and inertia of the models are required as input the data. The mass center and inertia moment were calculated considering the model structure with a uniformly distributed mass. These data are presented in Table 4.

Table 4. Mass and inertia of 1:50 floating models

\begin{tabular}{ccc}
\hline Model & Mass $[\mathrm{kg}]$ & $\begin{array}{c}\text { Moment of i } \\
\text { nertia }\left[\mathrm{kg} \cdot \mathrm{m}^{2}\right]\end{array}$ \\
\hline Square column & 198,3 & 94,8 \\
Circular column & 191,7 & 93,6 \\
\hline
\end{tabular}




\section{IMPACT OVER FLOATING STRUCTURE ANALYSIS}

Some of the validation regarding the motions of the free solid module implemented in the MPS simulation code used in the present study is presented at [9], for two degrees of feedom, with heave and roll motions.

At first, the results of the free floating motions are compared with that obtained by fixing the structures. Figure 9 shows the time series of wave runup in the fore column (column 1) and aft column (column 2) of the models with square and circular sectioned columns. In this section the results are analyzed only after the second impact, when the wave is completely developed.

Circular section column

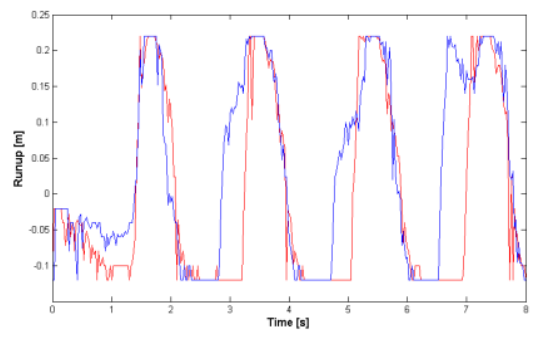

\section{Square section column}

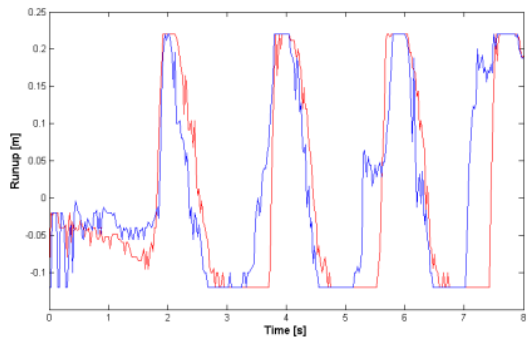

(a) Column 1
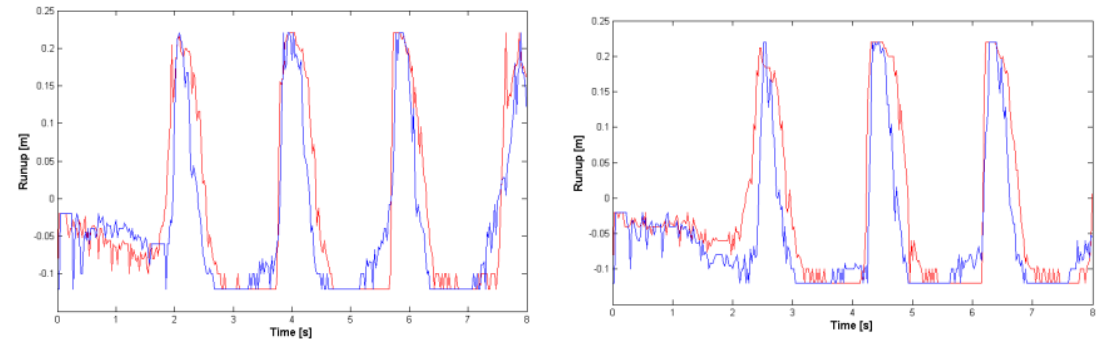

(b) Column 2

Figure 9. Wave runup obtained by MPS simulations of fixed structure (red) and free floating structure (blue) - Long wave

Figure 9 shows similar behaviors for the two models. In Figure 9a, the runup in the first column last longer for floating struture. At the second column, the opposite occurs, with shorter duration of wave runup, for the free motion cases. In the first column, the durations of wave runup of the squared section if shorter than the circular one, .

Figure 10 shows the comparison of pressure time histories between the square section column with the circular one considering the fixed and the free floating cases conditions. Pressure gauges are placed on both top and low ends of the columns. 


\section{Circular section column}

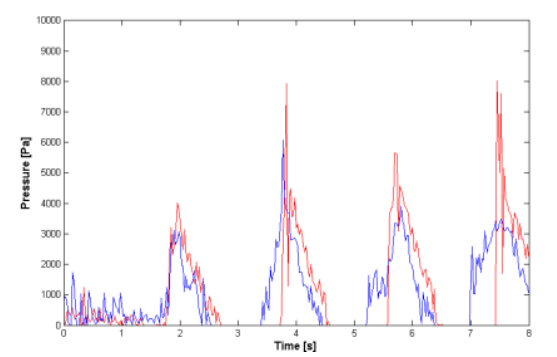

Square section column

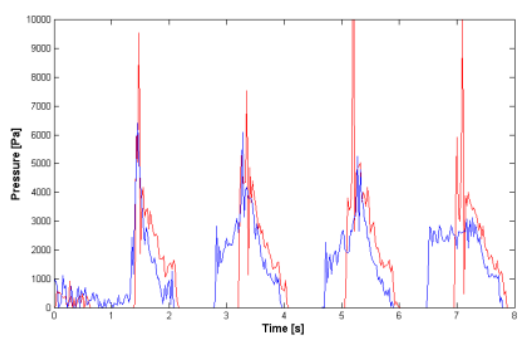

(a) Column 1- bottom sensor
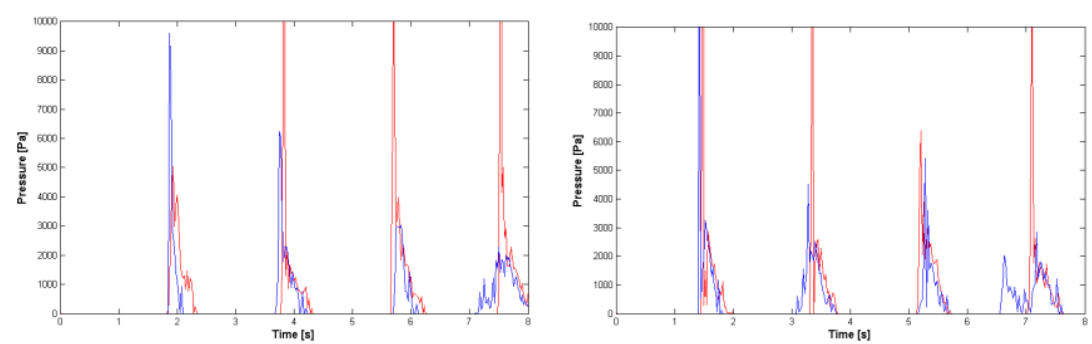

(b) Column 1 - top sensor
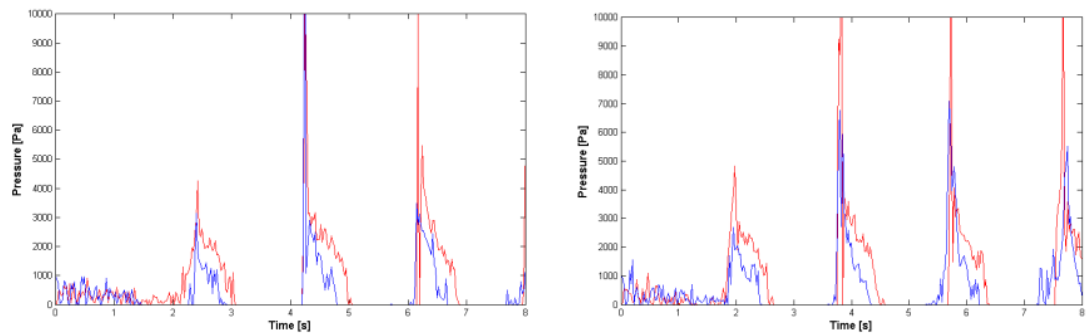

(c) Column 2- bottom sensor
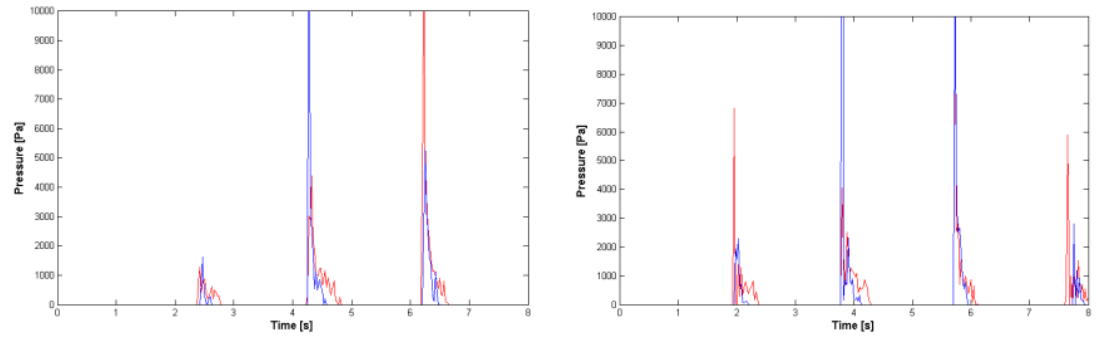

(d) Column 2 - top sensor

Figure 10. MPS calculated pressure time series - fixed structure (red) and floating structure (blue) - Medium wave

From Figure 10-a, for both models, the longer runup can be associated with the smaller pressures at the first column. The pressure sensor in the top of the fisrt column shows that the im- 
pulsive load at this region is overestimated when analysis is done considering fixed structure, as pointed out by Figure 10-b.

In the second columns, the main differences between the circular and squared models are regarding the impact at the top sensor. Due to the geometry of the first column on the incoming wave, a sequence of high impact pressure peaks occurs in the squared column.

In Figure 11 is shown the heave (translation in the $y$-axis - vertical) and pitch (the $z$-axis model rotation) motions considering the three different incident waves. The range of motion of the two structures is very close, the little difference in the movements is equivalent to the small difference in mass and moment of inertia between the two models presented in Table 4.
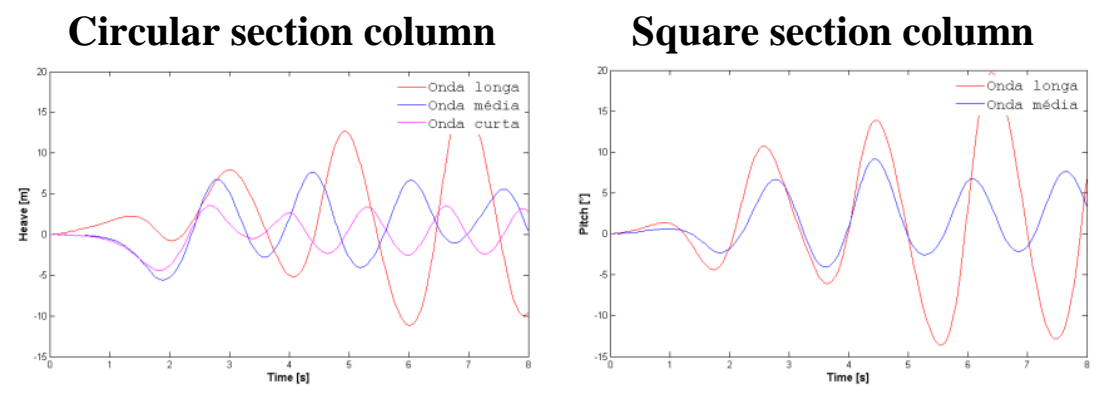

(a) pitch
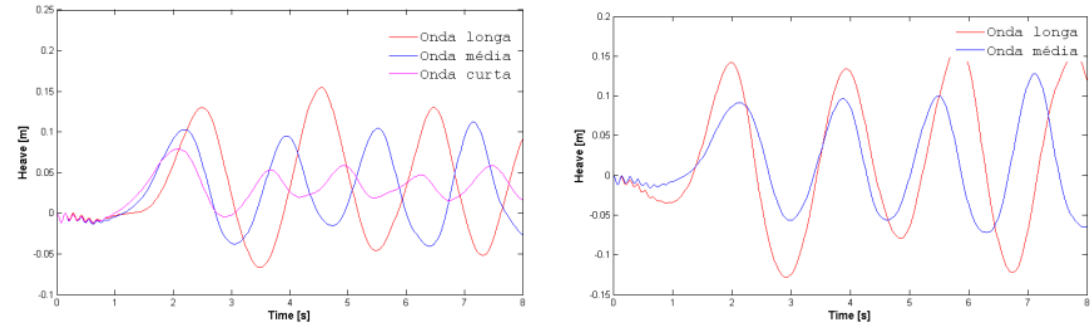

(b) heave

Figure 11. pitch e heave degrees of freedom model movements - long wave (red), medium wave (blue) and short wave (magenta)

Finally, the Figure 12 presents some snapshots of the first wave impact, for the model with circular section column (at left) and for model with square section column (at right) in case of the medium wave. The color gradient at the fluid indicates de velocity magnitude. The emergence of the pontoon of the model with circular sectioned column occurs at $t=2.2 \mathrm{~s}$, after the first impact on the fore column, because its displacement due to heave and pitching motions are slightly larger in the instant. 
Circular section column

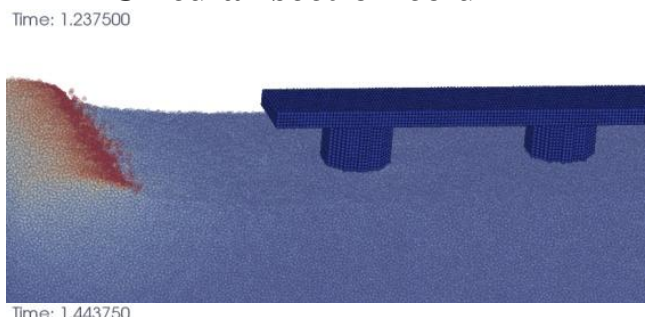

Time: 1.443750

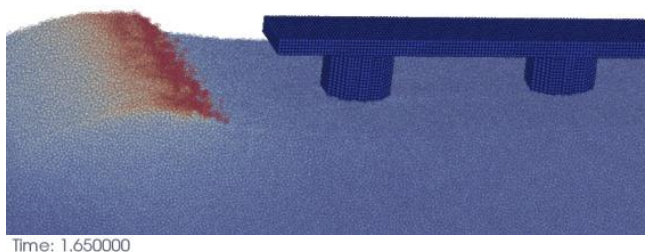

Time: 1.650000

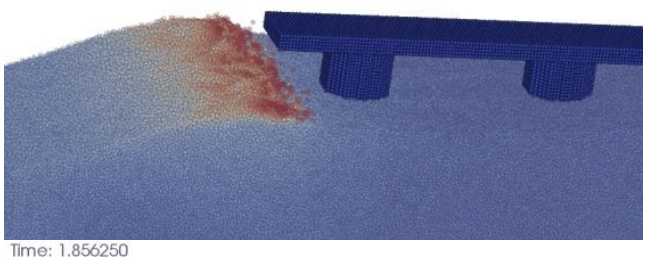

Time: 1.856250

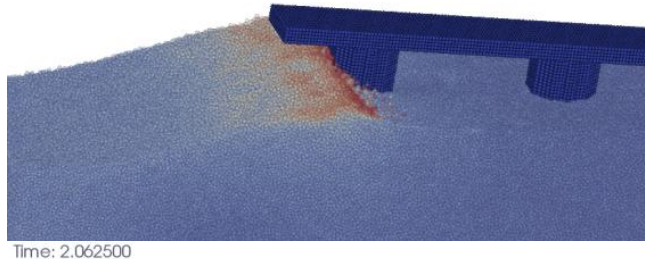

Time: 2.062500

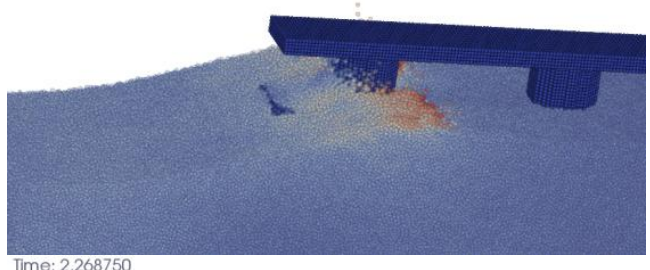

Time: 2.268750

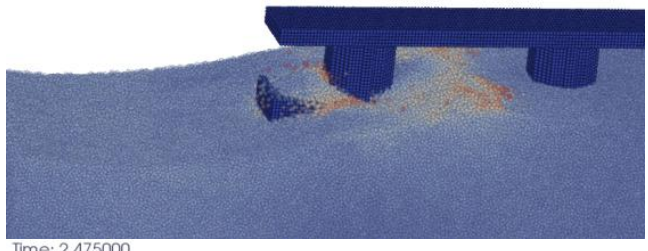

Time: 2.475000

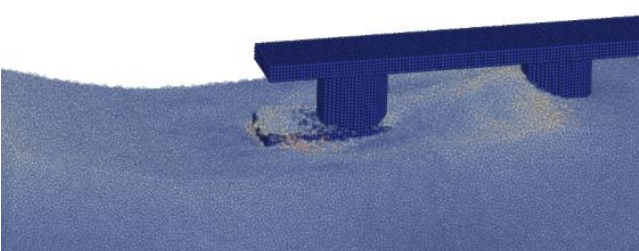

velocity magnitude $(\mathrm{m} / \mathrm{s}$

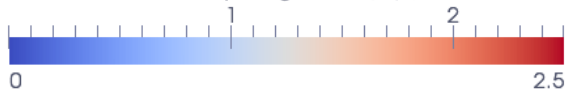

Figure 12. Snapshots of MPS simulation - Medium wave
Square section column
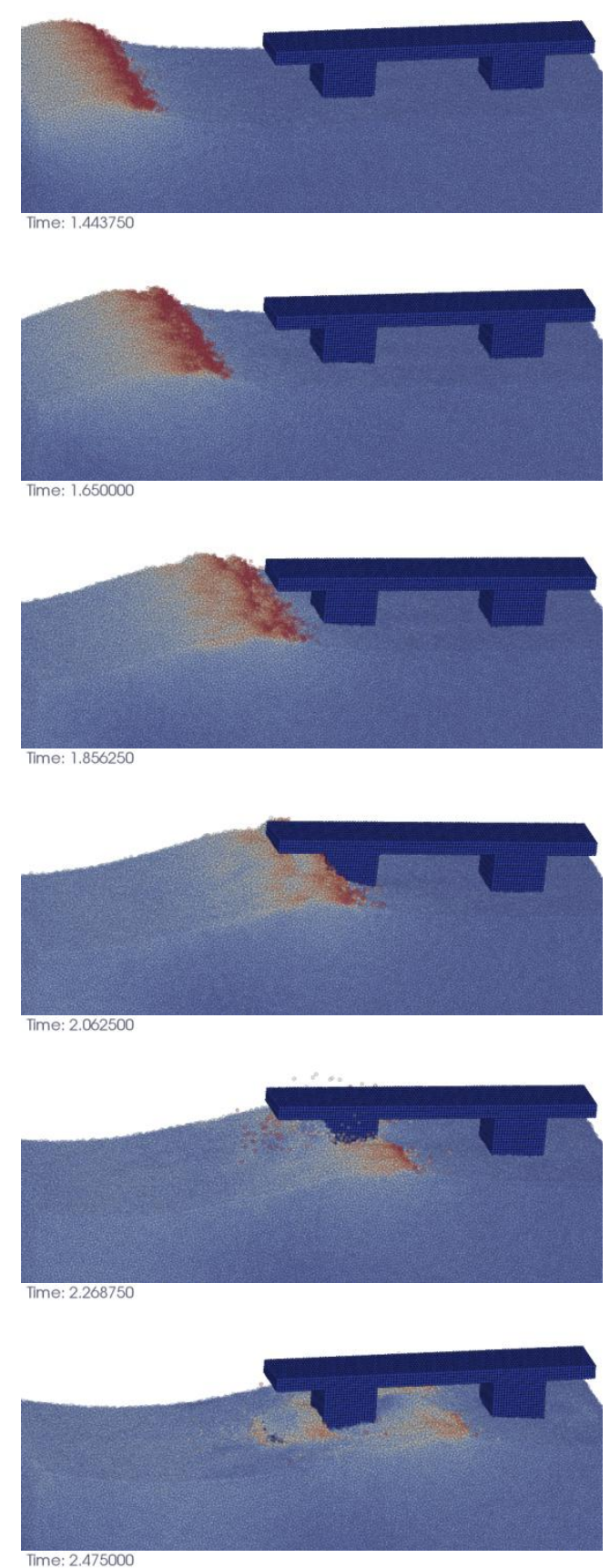

Time: 2.475000

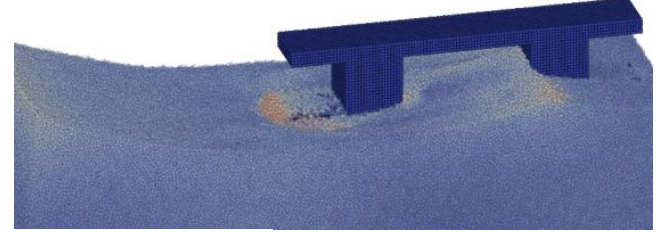
2.5 


\section{CONCLUSION}

This study is focused on non-linear nature phenomena resulting from the wave impact over offshore structures. Moving Particles Semi-Implicit (MPS) method, which is promising to fluid-structure interaction phenomena is applied for the simulation.

By comparing the results of the structures in fixed and free floating conditions, it is clear that the duration of the wave runup is shorter in the former one, and impact pressures in the fore column are overestimated. On the other hand, The duration of the wave runup in the model with circular sectioned column is in general longer than the squared one. The impact analysis with different column geometry models showed that the impact pressure on the top end of the second column of the model with square sectioned column is higher. Finally, the influence of the column geometry on the heavy and pitch motions in waves of different periods are also clarified, showing slightly large heavy and pitching motion in the model with square sectioned model.

The future development of this work comprises the application of distributed processing techniques, allowing the use of models with higher discretization and simulations takes less computational resources. Also comprises the development and aplication of different tools for modeling numerical tanks, such as reducing computational domain by the application of moving contours at the bottom of the tank, reducing the tank depth and maintaining the wave dynamics. For this initial stage we have satisfactory and quite encouraging for further investigation results.

\section{Acknowledgements}

The author would like to express their gratitude to PETROBRAS for financial support on the development of the MPS-based simulation code at the Numerical Offshore Tank (TPN-USP). The authors also would like extent their thanks to ANP by the financial support of the research.

\section{REFERENCES}

[1] R. Baarholm, "Experimental and Theoretical Study of Three-dimensional Effects on Vertical Wave-in-deck Forces," 30th International Conference on Ocean, Offshore and Artic Engineering, 2009.

[2] T. E. Kendon, C. Pakosdy, R. J. Baarholm, P. A. Berthelsen, C. Stansberg and S. Enger, "wave-in-deck impact: comparing CFD, simple methods, and model tests," in Proceedings of the ASME 2010 29th International Conference on Ocean, Offshore and 
Arctic Engineering, Shanghai, 2010.

[3] M. Sueyoshi, M. Kashiwagi and S. Naito, "Numerical Analysis of Wave Induced NonLinear Motions of a Two-Dimensional Floating Body by the Moving Particles SemiImplicit Methos," Journal of MArine Science and Technology, 13(2), pp. 85-98, 2008.

[4] B. Iwanowski, M. Lefranc and R. Wemmenhove, "CFD Simulation of Wave Run-up on a Semi-Submersible and Comparison with Experiment," Proceedings of the ASME 28th International Conference on Ocean, Offshore and Artic Engineering, 2009.

[5] A. Lal and M. Elangovan, "CFD Simulation and Validation of Flap Type Wave-Maker," World Academy of Sciences, Engineering and Technology, pp. 76-82, 2008.

[6] R. G. Dean and R. A. Dalrymple, Water Wave Mechanics, for Engineers and Scientists, Prentice Hall, 1984.

[7] C. A. Bellezi and L. Y. Cheng, "Análise do Impacto de Ondas no Convés Inferior de Plataformas Offshore por Método Moving Particles Semi-Implicit (MPS)," in IberianLatin American Congress on Computational Methods in Engineering, Ouro Preto, Brasil, 2011.

[8] C. A. Bellezi, F. A. Marcus, C. Liang-Yee and K. Nishimoto, "Análise de Hidrodinâmica Não-linear por Método MPS: Suavização da Flutuação de Pressão," $30^{\circ}$ Iberian-LatinAmerican Congress on Computational Methods in Engineering, 2009.

[9] M. M. Tsukamoto, L.-Y. Cheng and K. Nishimoto, "Numerical Study of the Motions in Shalow Water Waves of Floating Bodies Elastically Linked to the Bottom," in Proceedings of the ASME 28th International Conference on Ocean, Offshore and Artic Engineering, Honolulu, Hawaii, 2009.

[10] S. Koshizuka and Y. Oka, "A Particle Method for Complex Fluid Dynamics with Interfaces," in JSME Centennial Grand Congress, International Conference on Fluid Engineering, Tokyo, 1997.

[11] K. Shibata, S. Koshizuka and K. Tanizawa, "Three-dimensional Numerical Analysis of Shipping Water Onto a Moving Ship Using a Particle Method," Journal of Marine Science and Technology, pp. 214-227, 2009.

[12] K. Shibata and S. Koshizuka, "Numerical Analysis of Shipping Water Impact on a Deck Using a Particle Method," Ocean Engineering, vol. 34, p. 585-593, 2007.

[13] C. A. Bellezi, S. B. T. Mendes and L. Y. Cheng, "Estudo do Slamming em Cunha em V, Cilindro e Parábolas por Método Moving Particles Semi-Implicit (MPS)," in IberianLatin American Congress on Computational Methods in Engineering, Ouro Preto, Brasil, 2011. 